\title{
CLOSED IDEALS AND BI-ORTHOGONAL SYSTEMS IN RADICAL BANACH ALGEBRAS OF POWER SERIES
}

\author{
by MARC P. THOMAS
}

(Received 20th January 1981)

\section{Introduction}

In this paper we investigate the weighted convolution algebras $l^{p}\left(\omega_{n}\right)$, where $1 \leqq p<\infty$ and $\left\{\omega_{n}\right\}$ is a sequence of positive weights satisfying the following conditions. If $p=1$ we require $\omega_{0}=1, \omega_{n} \leqq 1$ and $\omega_{t+s} \leqq \omega_{t} \omega_{s}$. Then

$$
\left\|\sum_{k=0}^{\infty} \lambda_{k} e_{k}\right\|=\sum_{k=0}^{\infty}\left|\lambda_{k}\right| \omega_{k}
$$

If $1<p<\infty$ we require $\omega_{0}=1$ and

$$
\sup _{n}\left(\sum_{m=1}^{n-1}\left(\frac{\omega_{n}}{\omega_{n-m} \omega_{m}}\right)^{q}\right) \leqq 1,
$$

where $1 / p+1 / q=1$. Then

$$
\left\|\sum_{k=0}^{\infty} \lambda_{k} e_{k}\right\|=\left|\lambda_{0}\right|+\left(\sum_{k=1}^{\infty}\left(\left|\lambda_{k}\right| \omega_{k}\right)^{p}\right)^{1 / p}
$$

With these requirements, $\|f g\| \leqq\|f\|\|g\|$ where

$$
\left(\sum_{k=0}^{\infty} \lambda_{k} e_{k}\right)\left(\sum_{j=0}^{\infty} \mu_{j} e_{j}\right)=\sum_{m=0}^{\infty}\left(\sum_{j=0}^{m} \lambda_{m-j} \mu_{j}\right) e_{m}
$$

Finally, we will always require that $\left(\omega_{n}\right)^{1 / n} \rightarrow 0$ as $n \rightarrow \infty$. These conditions make $l^{p}\left(\omega_{n}\right)$ into commutative radical Banach algebra. We will also often write $e_{k}$ as $z^{k}$ and $\sum \lambda_{k} e_{k}$ as $\sum \lambda_{k} z^{k}$, treating it as an element in the algebra of formal power series $\mathbb{C}[[z]]$. If this is done, then the above convolution is simply multiplication of formal power series.

In all the following, $x$ will be a fixed element of $A=l^{p}\left(\omega_{n}\right)$ which is not a unit, i.e.

$$
x=\sum_{j=1}^{\infty} \zeta_{j} e_{j} \text { or } \quad x=\sum_{k=1}^{\infty} x_{k} e_{n(k)}, \quad x_{k} \neq 0
$$

We are interested in both the algebraic ideal $A x$ and the closed ideal $(A x)^{-}$generated by $x$. If $T$ is the linear operator of right translation on $l^{p}\left(\omega_{n}\right)$, then it is elementary that $(A x)^{-}=\overline{\operatorname{span}}_{n}\left\{T^{n} x\right\}$. 
Definition 1.1. Let $A=l^{p}\left(\omega_{n}\right)$ and let $K(s)=\left\{f \in A: f=\sum_{k=s}^{\infty} \lambda_{k} e_{k}\right\} . s=0,1,2, \ldots$ These are the standard ideals of $l^{p}\left(\omega_{n}\right)$. We say that a closed ideal $K$ is non-standard provided $K \neq K(s)$, all $s$.

It is well known that $(A x)^{-}$is standard if and only if $(A x)^{-}$contains a power of $z[2$, Lemma 4.5]. Certain criteria on $\left\{\omega_{n}\right\}$ are known which force $l^{p}\left(\omega_{n}\right)$ to have only standard ideals (see [2], [4] and [5]). These are generally conditions on $\left\{\omega_{n+1} / \omega_{n}\right\}$ which force $T$ to be a basis operator. At present it is still an open question whether there exist weights $\left\{\omega_{n}\right\}$ such that $A=l^{p}\left(\omega_{n}\right)$ contains non-standard ideals. There is an error in the commonly accepted construction in the literature [4, Theorem 5, p. 205]. We shall be concerned with the following five conditions:

Condition $I .(A x)^{-}$is a Schauder type ideal, and hence is non-standard (see Definition 1.2).

Condition II. $\sup _{n}\left\|x_{n}^{*}\right\|\left\|T^{n} x\right\|<\infty$, where $\left\{x_{n}^{*}\right\}$ are the bi-orthogonal functionals (see Definition 1.4).

Condition III. For some $l, \sup _{n}\left\|x_{n}^{*}\right\| \omega_{n+l}<\infty$ where $\left\{x_{n}^{*}\right\}$ are the bi-orthogonal functionals.

Condition IV. $(A x)^{-}$is standard but $z^{n} \notin A x$, all $n$.

Condition $V$. For some $l, z^{l} \in A x$ and hence $(A x)^{-}$is standard.

Condition I, which implies Condition II, is pertinent since the aforementioned construction in the literature [4, Theorem 4, p. 205] is an attempted, but erroneous, Schauder type ideal. In Section 2, we shall obtain necessary conditions for the existence of such Schauder type ideals (see Proposition 2.1, 2.2). Condition II is weaker than Condition I and such an ideal trivially satisfies Condition III (see Remark 4.6). Our major results occur in Section 3 and show that Condition III is very nearly equivalent to Condition $\mathrm{V}$ in the case that $(A x)^{-}$is standard, provided one considers equivalence classes of algebras (see Propositions 4.5, 4.7, and Theorem 4.9 for precise statements). However, if $(A x)^{-}$is a Schauder type ideal, and hence non-standard, it not only satisfies Condition III but also

$$
\sum_{n=0}^{\infty}\left\|x_{n}^{*}\right\| \omega_{n(1)+n}<\infty
$$

(see Proposition 2.2). Finally, Badé, Dales and Laursen [1] have given an example of an ideal either satisfying Condition IV or else non-standard.

Before proceeding to the proofs of the above statements, we list some preliminary definitions and results. As before we let $T f=z f, f \in l^{p}\left(\omega_{n}\right)$.

Definition 1.2. Let $A=l^{p}\left(\omega_{n}\right)$. Let $x=\sum_{k=1}^{\infty} x_{k} e_{n(k)}$ with $x_{k} \neq 0$. We say that $(A x)^{-}$is a Schauder type ideal if there exists a sequence of complex numbers $\left\{c_{m}\right\}_{m=0}^{\infty}$ and an 
increasing sequence of positive integers $\{l(m)\}_{m=0}^{\infty}$ satisfying the following two conditions:

1) $l(m+1)>l(m)+1$ for infinitely many $m$

2) $\sum_{m=0}^{\infty}\left\|\frac{T^{m} x}{c_{m}}-\frac{e_{l(m)}}{\omega_{l(m)}}\right\|<\infty$.

Such an ideal is necessarily non-standard [4, Lemma 4, p. 201], but we outline the argument for the sake of completeness. First note that $x$ generates a nonstandard ideal if and only if $T^{N} x$ generates a nonstandard ideal. Hence, by working with $T^{N} x$ instead of $x$ we may suppose the sum in (1.1) is less than one. Secondly, we may suppose that $c_{m}=x_{k(m)}$ where $n(k(m))+m=l(m)$, since $\left|x_{k(m)} \omega_{n(k)+m} c_{m}^{-1}-1\right|$ must tend to zero. If $\left.r_{m}=e_{l(m)} / \omega_{l(m)}\right)$, an element of the standard unit vector basis of $l^{p}\left(\omega_{n}\right)$, and if $s_{m}=\left(T^{m} x / x_{k(m)} \omega_{l(m)}\right)$, then (1.1) implies that $\left\{s_{m}\right\}$ is a perturbation of $\left\{r_{m}\right\}$ and consequently these are isomorphic basic sequences [3, Proposition 1.a.9]. Furthermore since there is a natural projection $P$ onto $\overline{\operatorname{span}}\left\{r_{m}\right\}$, it follows that $P$ restricted to $\overline{\operatorname{span}}\left\{s_{m}\right\}$ is an isomorphism. But $\overline{\operatorname{span}}\left\{s_{m}\right\}=(A x)^{-}$and if $(A x)^{-}$were standard it would have to be $K(n(1))$. The gaps in $\{l(m)\}$ resulting from condition 1$)$ in Definition 1.2 imply that $P$ restricted to $K(n(1))$ cannot be an isomorphism. This contradiction means $(A x)^{-}$must be nonstandard.

The reader may note that the second condition in Definition 1.2 could be relaxed a bit to read:

$$
\underset{m \rightarrow \infty}{\limsup }\left\|\frac{T^{m} x}{c_{m}}-\frac{e_{l(m)}}{\omega_{l(m)}}\right\|=0, \quad p=1
$$

or

$$
\sum_{m=0}^{\infty}\left\|\frac{T^{m} x}{c_{m}}-\frac{e_{l(m)}}{\omega_{l(m)}}\right\|^{q}<\infty, \quad 1<p<\infty, \quad \frac{1}{p}+\frac{1}{q}=1 .
$$

Most of our results would still hold but this does not seem to make a Schauder type ideal any easier to construct and we will not need such generality. Before considering Condition II we require the following lemma.

Lemma 1.3. Let $x \in l^{p}\left(\omega_{n}\right)$ and let $T$ be right translation on $l^{p}\left(\omega_{n}\right)$. Then

$$
T^{N} x \notin \overline{\operatorname{span}}\left\{T^{N} x\right\}, \text { all } N
$$

Proof. Suppose the result fails. Let $N$ be the least $N$ such that $T^{N} x \in \underset{\substack{n \neq N \\ n \neq n}}{ }\left\{T^{n} x\right\}$. Clearly $N>0$ since $x$ has a non-zero component at $n(1)$ and $T^{n} x$ vanishes at $n(1)$ if $n>0$. Let $X=\operatorname{span}_{0 \leqq k \leqq N-1}\left\{T^{k} x\right\}$ and $Y=\overline{\operatorname{span}}_{j \geqq N+1}\left\{T^{j} x\right\}$. Then

$$
\underset{n \neq N}{\operatorname{span}}\left\{T^{n} x\right\}=X \oplus Y,
$$


since $X$ is finite dimensional. So $T^{N} x=f+g, f \in X, g \in Y$. But if $f=\sum_{k=0}^{N-1} \alpha_{k} T^{k} x$ and if any $\alpha_{k}$ is non-zero this would force $T^{k} x \in \underset{n \neq k}{\operatorname{span}}\left\{T^{n} x\right\}$, contradicting the fact that $N$ was minimal. Hence $f=0$ and $T^{N} x=g \in Y$. But $g$ vanishes at $n(1)+N$ and $T^{N} x$ doesn't, a contradiction, and the result follows.

Definition 1.4. By Lemma 1.3, $\left\{T^{n} x\right\}$ is a minimal system in $\overline{\operatorname{span}_{n}}\left\{T^{n} x\right\}=(A x)^{-}$. Hence there are unique bi-orthogonal functionals $\left[3\right.$, p. 61] $\left\{x_{n}^{*}\right\}$ in $\left((A x)^{-}\right)^{*}$ satisfying $x_{n}^{*}\left(T^{m} x\right)=\delta_{n, m}$ and we say $\left(\left\{T^{m} x\right\},\left\{x_{n}^{*}\right\}\right)$ is a bi-orthogonal system.

In case $(A x)^{-}$is a Schauder type ideal, $\left\{T^{m} x\right\}$ is a basic sequence and $x_{n}^{*}\left(\sum_{m=0}^{\infty} \alpha_{m} T^{m} x\right)$ $=\alpha_{n}$. In this case $\left[3\right.$, p. 10], sup $\left\|x_{n}^{*}\right\|\left\|T^{n} x\right\|<\infty$ so Condition I implies Condition II. In general, though, a minimal system need not be a basic sequence $[3$, p. 62$]$ even if the supremum of the product of the respective norms is finite. In the next section we will derive certain necessary conditions for Condition I to hold.

\section{Schauder type ideals}

We first need to modify the notation of Definition 1.2. As noted before, by considering $T^{N} x$ instead of $x$ we may suppose the sum is less than one and that $c_{m}=x_{k(m)} \omega_{l(m)}$ where $n(k(m))+m=l(m)$. Let $I_{k-1}=\{m: n(k)+m=l(m)\}, k=1,2,3, \ldots$ Then we can rewrite (1.1) as

$$
\sum_{k=1}^{\infty} \sum_{m \in I_{k-1}}\left\|\frac{T^{m} x}{x_{k} \omega_{n(k)+m}}-\frac{e_{n(k)+m}}{\omega_{n(k)+m}}\right\|<1
$$

But $T^{m} x=x_{k} e_{n(k)+m}+\sum_{j \neq k} x_{j} e_{n(j)+m}$. Hence if $m \in I_{k-1}$ let

$$
\begin{aligned}
a(m) & =\left\|\sum_{j \neq k} \frac{x_{j} e_{n(j)+m}}{x_{k} \omega_{n(k)+m}}\right\| \\
& =\left[\sum_{j \neq k}\left|\frac{x_{j} \omega_{n(j)+m}}{x_{k} \omega_{n(k)+m}}\right|^{p}\right]^{1 / p} .
\end{aligned}
$$

Then (2.1) reads

$$
\sum_{k=1}^{\infty} \sum_{m \in I_{k-1}} a(m)<1 .
$$

In the erroneous example [4, Theorem 5, p. 205] in the literature $n(k)=2^{k}+1, k$ $=1,2,3, \ldots, I_{0}=\{0,1\}$ and $I_{k-1}=\left\{2^{k-1}, 2^{k-1}+1, \ldots 2^{k}-1\right\}, k=2,3,4, \ldots$. This means all $m$ in $I_{k-1}$ satisfy $m<n(k)$. We shall show that this is never possible for a Schauder type ideal.

Proposition 2.1. Let $x$ generate a Schauder type ideal with notation as above. Then the cardinality of $\left\{m: m \in I_{k-1}\right.$ and $\left.m \geqq n(k)\right\}$ is infinite. 
Proof. Suppose the result fails. Then there is $m_{0}$ such that $m \geqq m_{0}$ and $m \in I_{k-1}$ implies $m<n(k)$. Let $k(0)$ be a positive integer such that $n(k(0)) \geqq m_{0}$. Let $n(k(0))=h(0)$. There is $k(1)$ such that $n(k(0))=h(0) \in I_{k(1)-1}$, and $k(1)>k(0)$ necessarily. Continue by induction. If $k(j)$ has been chosen pick $k(j+1)$ with $n(k(j))=h(j) \in I_{k(j+1)-1}$. Let $y$ $=\sum_{j=0}^{\infty} x_{k(j)} e_{h(j)}$. Since $x \in l^{p}\left(\omega_{n}\right)$ and $\|y\| \leqq\|x\|$ it is clear that $y \in l^{p}\left(\omega_{n}\right)$ also. Since $l^{p}\left(\omega_{n}\right)$ is an algebra, $x y \in l^{p}\left(\omega_{n}\right)$ also and $x y=\sum_{j=0}^{\infty} x_{k(j)} T^{h(j)} x$. Since $\left\{e_{l(m)} / \omega_{l(m)}\right\}$ and $\left\{T^{m} x / x_{k(m)} \omega_{l(m)}\right\}$ are isomorphic basic sequences,

$$
\begin{aligned}
& \left\|\sum_{m=0}^{\infty} \alpha_{m} T^{m} x\right\|-\left\|\sum_{m=0}^{\infty} \alpha_{m} x_{k(m)} e_{l(m)}\right\| \\
& \geqq-\sum_{m=0}^{\infty}\left|\alpha_{m}\right|\left\|T^{m} x-x_{k(m)} e_{l(m)}\right\| \\
& \geqq-\sum_{m=0}^{\infty}\left|\alpha_{m} x_{k(m)}\right| \omega_{l(m)}\left\|\frac{T^{m} x}{x_{k(m)} \omega_{l(m)}}-\frac{e_{l(m)}}{\omega_{l(m)}}\right\| \\
& \geqq-\left[\sum_{m=0}^{\infty}\left(\mid \alpha_{m} x_{k(m)} \omega_{l(m)}\right)^{p}\right]^{1 / p}\left[\sum_{m=0}^{\infty} a(m)^{q}\right]^{1 / q} .
\end{aligned}
$$

Let $c=1-\sum_{m=0}^{\infty} a(m)>0$. Since the $q$-norm is less than the 1 -norm, the above implies:

$$
\left\|\sum_{m=0}^{\infty} \alpha_{m} T^{m} x\right\| \geqq c\left\|\sum_{m=0}^{\infty} \alpha_{m} x_{k(m)} e_{l(m)}\right\|
$$

in the case that $1<p<\infty$. The case $p=1$ is very similar in this and many other cases, hence we will often omit it. The above shows that

$$
\begin{aligned}
\|x y\|^{p} & =\left\|\sum_{j=0}^{\infty} x_{k(j)} T^{h(j)} x\right\|^{p} \\
& \geqq c^{p}\left\|\sum_{j=0}^{\infty} x_{k(j)} x_{k(j+1)} e_{h(j+1)+h(j)}\right\|^{p}
\end{aligned}
$$

since $n(k(j))=h(j) \in I_{k(j+1), 1}$, and the above

$$
=c^{p} \sum_{j=0}^{s}\left(\left|x_{k(j)} x_{k(j+1)}\right| \omega_{h(j+1)+h(j)}\right)^{p}
$$

Note that

$$
\frac{\left|x_{j}\right| \omega_{n(j)+m}}{\left|x_{k}\right| \omega_{h(k)+m}} \leqq a(m) \quad \text { if } \quad m \in I_{k-1}, \quad j \neq k
$$


Choose $1>d \geqq a(m)$ all $m$ using (2.2). Then

$$
\left|x_{k(j+1)}\right| \omega_{h(j+1)+h(j)} \geqq d^{-1}\left|x_{k(j-1)}\right| \omega_{h(j-1)+h(j)}
$$

for all $j \geqq 1$. Hence $(2.4)$ is

$$
\begin{aligned}
& \geqq c^{p} \sum_{j=1}^{\infty}\left(\left|x_{k(j)} x_{k(j+1)}\right| \omega_{h(j+1)+h(j)}\right)^{p} \\
& \geqq c^{p} d^{-p} \sum_{j=1}^{\infty}\left(\left|x_{k(j)} x_{k(j-1)}\right| \omega_{h(j-1)+h(j)}\right)^{p} \\
& \geqq c^{p} d^{-p} \sum_{j=0}^{\infty}\left(\left|x_{k(j+1)} x_{k(j)}\right| \omega_{h(j+1)+h(j)}\right)^{p}
\end{aligned}
$$

But $d^{-p}>1$, thus

$$
\sum_{j=0}^{\infty}\left(\left|x_{k(j+1)} x_{k(j)}\right| \omega_{h(j+1)+h(j)}\right)^{p}=\infty
$$

which forces $\|x y\|=\infty$, a contradiction.

As a consequence of this, one cannot build a Schauder type ideal upon a sequence like $\left\{2^{k}+1\right\}$. A bit more work, using the same recursive techniques as in the above proof would also show any geometric series is unsuitable. If there are any Schauder type ideals, they are either built upon somewhat lacunary sequences or upon sequences which oscillate between slow and fast growth. We finish this section with some other necessary conditions on a Schauder type ideal.

Proposition 2.2. Let $x$ generate a Schauder type ideal, $x=\sum_{k=1}^{\infty} x_{k} e_{n(k)}$ with notation as above. Then

(i) $z^{n(1)}\left((A x)^{-}\right) \subseteq A x$

(ii) $\sum_{n=0}^{\infty}\left\|x_{n}^{*}\right\| \omega_{n(1)+n}<\infty$, where $\left\{x_{n}^{*}\right\}$ are the bi-orthogonal functionals.

Proof. Let $y \in(A x)^{-}$. Then $y=\sum_{m=0}^{\infty} \alpha_{m} T^{m} x$ for some $\alpha_{m}$ 's since $\left\{T^{m} x\right\}$ is a basic sequence. Furthermore (2.3) above shows that $\sum_{m=0}^{\infty} \alpha_{m} x_{k(m)} e_{l(m)} \in l^{p}\left(\omega_{n}\right)$. Now since $\{l(m)\}$ is an increasing sequence (Definition 1.2) it follows that the cardinality of $I_{0}$ is finite. Hence for sufficiently large $m$

$$
\left|x_{1}\right| \omega_{n(1)+m} \leqq\left|x_{k(m)}\right| \omega_{l(m)}
$$

Thus $\sum_{m=0}^{\infty} \alpha_{m} x_{1} e_{n(1)+m}$ is in $l^{p}\left(\omega_{n}\right)$ also. Let $a=\left(x_{1}\right)^{-1} \sum_{m=0}^{\infty} \alpha_{m} x_{1} e_{n(1)+m}$. Then $a x$ $=\sum_{m=0}^{x} x_{m} T^{n(1)+m} x=z^{n(1)} y$. Since $y$ was arbitrary we have $z^{n(1)}\left((A x)^{-}\right) \subseteq A x$. For the second assertion note that there is $B<x$ such that $\left\|x_{n}^{*}\right\|\left\|T^{n} x\right\| \leqq B$ all $n$. Hence 
$\left\|x_{n}^{*}\right\| \leqq B /\left\|T^{n} x\right\|$. Let $r>\sup \left\{m: m \in I_{0}\right\}$. Then

$$
\begin{aligned}
\infty & >\sum_{m=r}^{\infty} a(m) \\
& \geqq \sum_{m=r}^{\infty}\left|\frac{x_{1} \omega_{n(1)+m}}{x_{k(m)} \omega_{l(m)}}\right| \\
& \geqq \sum_{m=r}^{\infty} \frac{\left|x_{1} \omega_{n(1)+m}\right|}{\left\|T^{n} x\right\|} \\
& \geqq \sum_{m=r}^{\infty}\left|\frac{1}{B}\left\|x_{m}^{*}\right\| x_{1} \omega_{n(1)+m}\right| .
\end{aligned}
$$

Thus $\sum_{m=0}^{\infty}\left\|x_{m}^{*}\right\| \omega_{n(1)+m}<\infty$, completing the proof of the proposition.

It is rather surprising that the assertions of Proposition 2.2 for a Schauder type (hence non-standard) ideal are similar to later conditions we will use to determine when $A x$ or an equivalent algebra contains a power of $z$ in the case $(A x)^{-}$is standard (see Theorem 4.9). This casts some more doubt upon the question whether Schauder type ideals exist or not.

\section{Equivalent algebras}

Let $A=l^{p}\left(\omega_{n}\right)$ and $x=\sum_{k=1}^{\infty} x_{k} e_{n(k)}$ as before.

Definition 3.1. Let $A_{1}=l^{p}\left(\tilde{\omega}_{n}\right)$. We say that $A_{1}$ is equivalent to $A=l^{p}\left(\omega_{n}\right)$ if there exists a constant $C>0$ such that

$$
e^{-C_{n}} \leqq \frac{\tilde{\omega}_{n}}{\omega_{n}} \leqq e^{C_{n}}, \quad \text { all } n
$$

and we write $A_{1} \sim A$. We say that $A_{1}$ is strongly equivalent to $A$ if there exists a real constant $c$ such that $\tilde{\omega}_{n}=e^{-c n} \omega_{n}$, all $n$, and we write $A_{1} \approx A$. Clearly strong equivalence implies equivalence. Finally, if $x \in A_{1}$ also, then we write $A_{1} \tilde{x} A$ if $A_{1} \sim A$ and $A_{1} \tilde{\tilde{x}} A$ if $A_{1} \approx A$. All these relations are clearly equivalence relations.

If $A_{1} \approx A$ it is clear they are isomorphic under the mapping

$$
\theta\left(\sum_{n=0}^{\infty} \alpha_{n} e_{n}\right)=\sum_{n=0}^{\infty} \alpha_{n} e^{c n} e_{n}
$$

from $A=l^{p}\left(\omega_{n}\right)$ to $A_{1}=l^{p}\left(e^{-c n} \omega_{n}\right)$. Hence there is a one to one correspondence between their non-standard ideals, if any exist. It is also clear there is an inclusion map from $A$ to $A_{1}$ provided $c \geqq 0$. However, in general if $c<0$ and $a \in A$ then $z^{l} a$ need not be in $A_{1}$ 
for any $l$. One question still unanswered is whether $(A x)^{-}$standard and $A \tilde{x} A_{1}$ implies $\left(A_{1} x\right)^{-}$standard. Of course this is clear if $\tilde{\omega}_{n} \leqq \omega_{n}$, but the general case is unresolved. In the next section we investigate the relationship between Condition III and Condition V.

\section{The algebraic ideal generated by $x$}

We continue the notation of the previous sections. Let $x=\sum_{k=1}^{\infty} x_{k} e_{n(k)}=\sum_{j=n(1)}^{\infty} \zeta_{j} e_{j}$, so $\zeta_{n(k)}=x_{k}$. Let $\left\{e_{n}^{*}\right\}$ be the dual basic sequence of $l^{p}\left(\omega_{n}\right)^{*}=l^{q}\left(1 / \omega_{n}\right)$, i.e. $e_{n}^{*}\left(e_{m}\right)=\delta_{n, m}$. In this section we will often give the proofs for $1<p<\infty$ when the case $p=1$ is similar. We now define the sequence $\left\{c_{n}\right\}$.

Definition 4.1. Let $c_{0}=1 / x_{1}$. If $c_{0}, c_{1}, c_{2}, \ldots, c_{n-1}$ have been chosen let

$$
\begin{aligned}
c_{n} & =\frac{-1}{x_{1}} \sum_{i=0}^{n-1} c_{i} \zeta_{n(1)+n-i} \\
& =\frac{-1}{x_{1}} \sum_{k=1}^{n} c_{n-k} \zeta_{n(1)+k} .
\end{aligned}
$$

It is routine to check the following properties of $\left\{c_{n}\right\}$.

Lemma 4.2. Let $\left\{c_{n}\right\}$ be chosen as above. Then:
(i) $\sum_{n=0}^{N} c_{n} T^{n} x=z^{n(1)}$ on $[n(1), N+n(1)]$.
(ii) $\left(\sum_{n=0}^{n} c_{n} z^{n}\right) x=z^{n(1)}$ as formal power series in $\mathbb{C}[[z]]$.
(iii) If $\chi_{m}^{*}=\sum_{n=0}^{m} c_{n} e_{n(1)+m-n}^{*}$ in $l^{q}\left(1 / \omega_{n}\right)$, then $\chi_{m}^{*}\left(T^{n} x\right)=\delta_{n, m}$.

(iv) If $(A x)^{-}$is standard $x_{n}^{*}=\chi_{n}^{*}$.

Proof. Assertion (i) is easily established using equation (4.1) and (iii) is easily established using equation (4.2). It is elementary that (i) implies (ii) and (iii) implies (iv).

We need two more preliminary lemmas.

Lemma 4.3. If $(A x)^{-}$is standard, if $l \geqq n(1)$, and if $\left\|\left.x_{n}^{*}\right|_{K(l)}\right\| \omega_{n+l} \leqq B_{n}$ each $n$. Then $\left\|x_{n}^{*}\right\| \omega_{n+l+(l-n(1))} \leqq B_{n+(l-n(1))}$ each $n$.

Proof. Since $(A x)^{-}$is standard $\left((A x)^{-}\right)^{*}=l^{q}\left([n(1), \infty), 1 / \omega_{n}\right)$ and by Lemma 4.2

$$
\left\|x_{n}^{*}\right\|=\sup _{n(1) \leqq k \leqq n(1)+n} \frac{\left|c_{n(1)+n-k}\right|}{\omega_{k}} \text { if } p=1
$$


and

$$
\left\|x_{n}^{*}\right\|^{q}=\sum_{k=n(1)}^{n(1)+n}\left|\frac{c_{n(1)+n-k}}{\omega_{k}}\right|^{q} \quad \text { if } \quad 1<p<\infty, \quad \frac{1}{p}+\frac{1}{q}=1
$$

We assume $1<p<\infty$ since the case $p=1$ is very similar. Letting $k=n(1)+r-l$,

$$
\begin{aligned}
\left\|x_{n}^{*}\right\|^{q} & =\sum_{r=1}^{n+1}\left(\frac{\left|c_{n+l-r}\right|}{\omega_{n(1)+r-1}}\right)^{q} \\
& =\sum_{r=1}^{n+l}\left(\frac{\left|c_{n+l-r}\right|}{\omega_{r}} \frac{\omega_{r}}{\omega_{n(1)+r-l}}\right)^{q} .
\end{aligned}
$$

Since $n(1)+r-l<r$ the above is

$$
\begin{aligned}
& \leqq \sum_{r=l}^{n+l}\left(\frac{c_{n+l-r}}{\omega_{r}}\right)^{q} \\
& =\left\|\left.x_{n+l-n(1)}^{*}\right|_{K(l)}\right\|^{q} \\
& \leqq\left(B_{n+(l-n(1))} / \omega_{(n+l-n(1))+l}\right)^{q} .
\end{aligned}
$$

Thus

$$
\left\|x_{n}^{*}\right\| \omega_{n+l+(l-n(1))} \leqq B_{n+(l-n(1))}
$$

Lemma 4.4. Let $A=l^{p}\left(\omega_{n}\right)$. If for some $l \geqq n(1), z^{l} \in A x$, then

$$
z^{l}\left((A x)^{-}\right) \subseteq A x
$$

Proof. If $a x=z^{l}$ and $b \in(A x)^{-}$then $z^{l} b=a x b=(a b) x$ and the result follows.

Contrast this with the case of a Schauder type ideal (Proposition 2.2) where $z^{n(1)}\left((A x)^{-}\right) \subseteq A x$ even though $z^{l} \notin(A x)^{-}$, all $l$.

We now have the following.

Proposition 4.5. Suppose $z^{l} \in A x$ for some $l$ (which is necessarily greater than or equal to $n(1))$. Then
(i) $\sup _{n}\left\|\left.x_{n}^{*}\right|_{K(l)}\right\| \omega_{n+1}<\infty$
(ii) $\sup _{n}\left\|x_{n}^{*}\right\| \omega_{n+2 l-n(1)}<\infty$
(iii) $z^{l}\left((A x)^{-}\right) \subseteq A x$. 
Proof. Note $(A x)^{-}$is standard and equals $K(n(1))$. Clearly (ii) follows from (i) by Lemma 4.3 and (iii) follows by Lemma 4.4 . We will prove (i) in the case $1<p<\infty$ since the case $p=1$ is similar.

$$
\begin{aligned}
\left\|\left.x_{n}^{*}\right|_{K(l)}\right\|^{q} & =\sum_{k=1}^{n(1)+n}\left(\frac{\left|c_{n(1)+n-k}\right|}{\omega_{k}}\right)^{q} \\
& =\sum_{k=l}^{n(1)+n}\left(\frac{\left|x_{n}^{*}\left(z^{k}\right)\right|}{\omega_{k}}\right)^{q}
\end{aligned}
$$

Now $z^{k}=z^{k-l} z^{l}=z^{k-l} a x$ where $a x=z^{l}$. Let $a=\sum_{j=0}^{\infty} \lambda_{j} z^{j}$ and by continuity of multiplication $a x=\sum_{j=0}^{\infty} \lambda_{j} T^{j} x=z^{l}$. Hence $z^{k}=\sum_{j=0}^{\infty} \lambda_{j} T^{j+k-l} x$. So $x_{n}^{*}\left(z^{k}\right)=\lambda_{j}$ where $j+k$ $-l=n$ i.e. $j=n+l-k$. Hence $x_{n}^{*}\left(z^{k}\right)=\lambda_{n+l-k}$, and from (4.3) it follows that

$$
\begin{aligned}
\|\left. x_{n}^{*}\right|_{K(l)}||^{q} \omega_{n+l}^{q} & =\sum_{k=l}^{n(1)+n}\left(\frac{\left|\lambda_{n+l-k}\right| \omega_{n+l}}{\omega_{k}}\right)^{q} \\
& =\sum_{k=l}^{n(1)+m}\left(\frac{\left|\lambda_{n+l-k}\right| \omega_{n+l-k} \omega_{n+l}}{\omega_{n+l-k} \omega_{k}}\right)^{q} \\
& \leqq \sum_{k=l}^{n(1)+n}\left(\frac{\|a\| \omega_{n+l}}{\omega_{n+l-k} \omega_{k}}\right)^{q} .
\end{aligned}
$$

This is less than $\|a\|$ if $l>n(1)$. If $l=n(1)$ then it is still less than $2\|a\|$. In any case it is bounded and the result follows.

We now seek a result in the converse direction. We wish to first note the following.

Remark 4.6. If $\sup _{n}\left\|x_{n}^{*}\right\|\left\|T^{n} x\right\|<\infty$, then since $\left\|T^{n} x\right\| \geqq\left|x_{1}\right| \omega_{n(1)+n}$ it easily follows that $\sup _{n}\left\|x_{n}^{*}\right\| \omega_{n(1)+n}<\infty$, also.

Proposition 4.7. Let $A=l^{p}\left(\omega_{n}\right)$ and suppose $(A x)^{-}$is standard. If $l \geqq n(1)$ and

$$
\sup _{n}\left\|\left.x_{n}^{*}\right|_{K(l)}\right\| \omega_{n+l}<\infty
$$

then there is $A_{1} \tilde{\tilde{x}} A$ such that $z^{2 l} \in A_{1} x$. More generally, the same conclusion holds if

$$
\left.\sup _{n}|| x_{n}^{*}\right|_{K(l)}|| \omega_{n+l} e^{-k n}<\infty
$$

for some $l \geqq n(1)$ and $k>0$. 
Proof. $(A x)^{-}=K(n(1))$. If $n(1)+n \geqq l$ then

$$
\frac{\left|c_{n(1)+n-t}\right|}{\omega_{l}} \leqq\left\|\left.x_{n}^{*}\right|_{K(t)}\right\|, \quad 1 \leqq p<\infty
$$

and hence

$$
\sup _{n \geqq l-n(1)}\left|\frac{c_{n(1)+n-l} \omega_{n+l}}{\omega_{l}}\right|^{p}<\infty
$$

or

$$
\sup _{n \geqq l-n(1)}\left|\frac{c_{n(1)+n-l} \omega_{n+l}}{\omega_{l}}\right|^{p} e^{-k n}<\infty
$$

depending upon which hypothesis holds. In any case we can choose $\tilde{\omega}_{n}=\omega_{n} e^{-c n}, c>0$, such that:

$$
\sum_{n=i-n(1)}^{\infty}\left|c_{n(1)+n-1}\right|^{p}\left(\tilde{\omega}_{n+l}\right)^{p}<\infty
$$

If (4.4) holds any $c>0$ will suffice but $c=0$ may not.

Let $a=\sum_{n=l-n(1)}^{\infty} c_{n(1)+n-l} z^{n+l} \in l^{p}\left(\tilde{\omega}_{n}\right)=A_{1}$. Clearly $A_{1} \approx A, x \in A_{1}$ also since $c>0$, and

$$
\begin{gathered}
a=z^{2 l-n(1)} \sum_{n=l-n(1)}^{\infty} c_{n(1)+n-l^{2}} z^{n(1)+n-l} \\
n-n\left(11 \sum_{k=0}^{\infty} r .7^{k}\right.
\end{gathered}
$$

as formal power series in $\mathbb{C}[[z]]$. Hence by Lemma $4.2 a x=z^{21-n(1)} z^{n(1)}=z^{21}$ as formal power series, but since $a$ and $x$ are both in $A_{1}$ this implies $z^{2 l} \in A_{1} x$, and the result follows.

Proposition 4.7 suggests that we consider the problem of when a power of $z$ is in $A x$ not simply in $A$, but in the class of algebras equivalent to $A$.

Definition 4.8. Let $x \in A=l^{p}\left(\omega_{n}\right)$. We say $\left\{\left\|x_{n}^{*}\right\|\right\}$ is of exponential type if for some $c$ and $l$

$$
\sup _{n}\left\|x_{n}^{*}\right\| \omega_{n+l} e^{-c n}<\infty
$$

where $\left\|x_{n}^{*}\right\|$ is the norm of the bi-orthogonal functional $x_{n}^{*}$ in $\left((A x)^{-}\right)^{*}$. 
A couple of remarks are in order in the case $(A x)^{-}$is standard. First by Lemma 4.3 equation (4.6) is equivalent to

$$
\sup _{n}\left\|\left.x_{n}^{*}\right|_{K(m)}\right\| \omega_{n+m} e^{-k n}<\infty
$$

for some $k$ and $m$. Secondly suppose $A_{1} \tilde{x} A$ and both $(A x)^{-}$and $\left(A_{1} x\right)^{-}$are standard. Then if (4.6) holds for $x$ in $A$ it also holds for $x$ in $A_{1}$ even though the norm $\left\|x_{n}^{*}\right\|_{1}$ of $x_{n}^{*}$ as an element of $\left(\left(A_{1} x\right)^{-}\right)^{*}$ is different from its norm $\left\|x_{n}^{*}\right\|$ in $\left((A x)^{-}\right)^{*}$. This is a consequence of the following computation:

$$
\begin{aligned}
\left(\left\|x_{n}^{*}\right\|_{1} \tilde{\omega}_{n+l}\right)^{q} & =\sum_{k=n(1)}^{n(1)+n}\left|\frac{c_{n(1)+n-k}}{\tilde{\omega}_{k}}\right|^{q}\left(\tilde{\omega}_{n+l}\right)^{q} \\
& =\sum_{k=n(1)}^{n(1)+n}\left(\frac{\omega_{k}}{\tilde{\omega}_{k}}\left|\frac{c_{n(1)+n-k}}{\omega_{k}}\right|\right)^{q}\left(\frac{\tilde{\omega}_{n+l}}{\omega_{n+l}}\right)^{q}\left(\omega_{n+l}\right)^{q} \\
& \leqq \sum_{k=n(1)}^{n(1)+n}\left(e^{c k}\left|\frac{c_{n(1)+n-k}}{\omega_{k}}\right|\right)^{q}\left(e^{C(n+l)} \omega_{n+l}\right)^{q}
\end{aligned}
$$

by $(3.1)$

$$
\begin{aligned}
& \leqq\left(\sum_{k=n(1)}^{n(1)+n}\left|\frac{c_{n(1)+n-k}}{\omega_{k}}\right|^{q}\right)\left(\omega_{n+l}\right)^{q}\left(e^{2 C n} e^{2 C l}\right)^{q} \\
& =\left(\left\|x_{n}^{*}\right\| \omega_{n+l}\right)^{q}\left(e^{2 C n} e^{2 C l}\right) .
\end{aligned}
$$

Thus Definition 4.8. does not depend upon whether we regard $x$ as an element of $A$ or $A_{1}$ provided $A \tilde{x} A_{1}$ and both $(A x)^{-}$and $\left(A_{1} x\right)^{-}$are standard. We now have our major result.

Theorem 4.9. Let $A=l^{p}\left(\omega_{n}\right), x \in A$ and suppose $(A x)^{-}$is standard. Then the following are equivalent:

(1) There is $A_{1} \tilde{\tilde{x}} A$ with $A_{1} x$ containing a power of $z$.

(2) There is $A_{1} \tilde{x} A$ with $A_{1} x$ containing a power of $z$.

(3) $\left\{\left\|x_{n}^{*}\right\|\right\}$ is of exponential type.

(4) There is $A_{1} \tilde{x} A$ with $\left(A_{1} x\right)^{-}$standard and $z^{l}\left(\left(A_{1} x\right)^{-}\right) \subseteq A_{1} x$, for some l.

Proof. If (1) holds clearly (2) does also since strong equivalence implies equivalence. If (2) holds it is clear that $\left(A_{1} x\right)^{-}$is standard. Proposition 4.5 implies that

$$
\sup _{n}\left\|x_{n}^{*}\right\|_{1} \tilde{\omega}_{n+L}<\infty
$$


for some $L$, where $A_{1}=l^{p}\left(\tilde{\omega}_{n}\right)$. But our remark above implies that

$$
\sup _{n}\left\|x_{n}^{*}\right\| \omega_{n+L} e^{-r n}<\infty
$$

for some $r$ and thus (3) holds. If (3) holds Proposition 4.7 implies there is $A_{1} \tilde{x} A$ with $z^{l} \in A_{1} x$, some $l>n(1)$. Then Lemma 4.4 implies $z^{l}\left(\left(A_{1} x\right)^{-}\right) \subseteq A_{1} x$. This proves (4) since $\left(A_{1} x\right)^{-}$is clearly standard. Finally if (4) holds $z^{n(1)} \in\left(A_{1} x\right)^{-}$and hence $z^{i+n(1)} \in A_{1} x$. Since $A_{1} \tilde{x} A$ we can find $C>0$ such that

$$
e^{-C n} \leqq \frac{\tilde{\omega}_{n}}{\omega_{n}} \leqq e^{C n} \quad \text { all } \quad n
$$

Then $A_{2}=l^{P}\left(e^{-C n} \omega_{n}\right)$ is strongly equivalent to $A, A_{2} \supseteq A_{1}$ so $x \in A_{2}$ and $z^{l+n(1)} \in A_{2} x$ also. Thus (1) holds, completing the proof of the theorem.

\section{REFERENCES}

1. W. G. Bade, H. G. Dales and K. B. Laursen, paper to appear in Journal of Functional Analysis.

2. S. Grabiner, Weighted shifts and Banach algebras of power series, American J. Math. 97 (1975), 16-42.

3. J. Lindenstrauss and L. Tzafriri, Classical Banach Spaces, Vol. 1.

4. N. K. Nixolskil, Selected problems of weighted approximation and spectral analysis, Proc. Steklov Inst. Math. 120 (1974) (AMS translation, 1976).

5. A. L. SHields, Weighted shift operators and analytic function theory, Topics in Operator Theory (ed. C. Pearcy) (AMS Surveys, No. 13, Providence, RI, 1974).

California State College

9001 Stockdale Highway

BakeRsfield CA 93309 